\title{
Decreasing Trend in Incidence of Late Onset Culture Positive Bloodstream Infections but Not Late Onset Meningitis in Preterm Infants $<33$ Weeks Gestation in Canadian Neonatal Intensive Care Unit
}

\author{
Qi Zhou ${ }^{a, b}$ Melissa Ong ${ }^{c}$ Marie Lan ${ }^{d}$ Xiang Y. Ye ${ }^{b}$ Joseph Y. Ting ${ }^{e, f}$ \\ Prakesh S. Shah ${ }^{b}$ Shoo K. Lee ${ }^{b}$ on behalf of the Canadian Neonatal Network \\ (CNN) Investigators \\ aDepartment of Neonatology, Children's Hospital of Fudan University, Shanghai, China; ${ }^{\mathrm{b}}$ Department of Pediatrics, \\ Mount Sinai Hospital and University of Toronto, Toronto, ON, Canada; 'Department of Acute Medicine, Lewisham \\ and Greenwich Trust, London, UK; dSchulich School of Medicine \& Dentistry, Western University, London, ON, \\ Canada; 'Department of Pediatrics, University of British Columbia, Vancouver, BC, Canada; fDepartment of \\ Pediatrics, University of Alberta, Edmonton, AB, Canada
}

\section{Keywords}

Preterm infant · Late onset sepsis - Late onset meningitis · Intraventricular hemorrhage

\begin{abstract}
Introduction: Recent studies reported decreased incidence of late onset sepsis in the neonatal intensive care unit (NICU), but it is unclear whether this is also true for late onset meningitis. Recent reports that both meningitis and intraventricular hemorrhage (IVH) are associated with systemic inflammation also raise questions about an association between the 2. Methods: All preterm infants $<33$ weeks gestational age admitted to CNN NICUs from 2010 to 2018 were included. We compared incidence trends of late onset culture positive bloodstream infection (CPBSI) and late onset meningitis, and examined the association of meningitis and IVH (exposure), after adjustment for potential confounders. Results: Of 36,573 infants included, 32,198 had no infection, 3,977
\end{abstract}

had only late onset CPBSI and 398 had late onset meningitis. There was significant decrease in incidence of late onset CPBSI (14\%-10\%; adjusted odds ratio (AOR) $=0.93 ; 95 \%$ confidence interval $[\mathrm{Cl}] 0.92,0.95)$ but not late onset meningitis (1.6\%-1.2\%; AOR $=0.98 ; 95 \% \mathrm{Cl} 0.94,1.01)$. Compared to infants with no IVH grade 3 or above, infants with IVH grade 3 , or above had higher odds of late onset meningitis versus no infection (AOR 4.16; 95\% Cl 3.17, 5.44), and higher odds of late onset meningitis versus late onset CPBSI (AOR 4.11;95\% $\mathrm{Cl} 3.08,5.50)$. Conclusions: There was a decreasing trend of late onset CPBSI but not late onset meningitis. An association between late onset meningitis and IVH grade 3 or above was observed. Late onset CPBSI and meningitis may have different risk factors and require different prevention strategies.

(C) 2021 The Author(s).

Published by S. Karger AG, Basel

A full list of CNN Investigators is provided in the Author Contributions. karger@karger.com www.karger.com/neo

Karger $\stackrel{\text { ' }}{5}$

BOPEN ACCESS
(C) 2021 The Author(s)

Published by S. Karger AG, Basel

This is an Open Access article licensed under the Creative Commons Attribution-NonCommercial-4.0 International License (CC BY-NC) (http://www.karger.com/Services/OpenAccessLicense), applicable to the online version of the article only. Usage and distribution for commercial purposes requires written permission. 


\section{Introduction}

Sepsis is a leading cause of mortality and morbidity among preterm infants $<33$ weeks' gestational age (GA) in the neonatal intensive care unit (NICU), and meningitis is usually considered a subtype of systemic sepsis [13]. However, the Canadian Neonatal Network (CNN) made a preliminary observation that while the incidence of nosocomial infection had decreased in recent years, there was no change in the incidence of meningitis $[4,5]$. Additionally, there have been reports that both meningitis and intraventricular hemorrhage (IVH) are associated with systemic inflammation, and may promote excitotoxic parenchymal damage in the brain $[6,7]$. Since both these conditions occur during the neonatal period and may share a common pathophysiological pathway, we speculated that there may be a relationship between the 2 and hypothesized that IVH may be associated with meningitis in preterm infants. Our aims were to examine whether there were differences in incidence trends for late onset sepsis and meningitis among preterm infants $<33$ weeks GA in Canadian NICUs, and whether there was an association between meningitis and IVH.

\section{Methods}

\section{Study Design and Population}

We conducted a retrospective cohort study of all preterm infants $<33$ weeks GA who were admitted to tertiary-level NICUs participating in the CNN from January 1, 2010, to December 31, 2018. We excluded infants who were moribund on admission, with major congenital anomalies, missing birth date, culture-proven early onset sepsis, or meningitis. Patients with late onset culture positive bloodstream infection (CPBSI) or late onset meningitis (cerebrospinal fluid culture positive, with or without CPBSI) were identified.

\section{Data Collection}

The CNN database prospectively collects information on maternal pregnancy and delivery, infant characteristics, outcomes, and resource use from all 30 tertiary-level NICUs in Canada, and has high reliability and internal consistency [8]. At participating sites, trained abstractors' abstract data from patient charts using a standardized manual of data definitions into an electronic data-set with built-in error checking was analyzed and validated by separate audit [9]. Ethics approval and waiver of need for consent were granted at each site by either the local Research Ethics Board or through an institutional quality improvement process for data collection and benchmarking.

\section{Study Variables and Definitions}

Enrolled infants were classified into 3 groups: no infection, late onset CPBSI only, and late onset meningitis (with or without late onset CPBSI). Late onset CPBSI in the current study was defined as at least 1 positive blood culture obtained from a neonate clinically suspected of having infection after 2 calendar days of life, as defined by the CNN manual of definitions [9]. Late onset meningitis was defined by at least 1 positive cerebrospinal fluid culture obtained from a neonate clinically suspected of infection after 2 calendar days of life, with or without late onset CPBSI. When potential skin flora like Coagulase negative staphylococcus (CoNS) was isolated from the blood or cerebrospinal fluid sample, it would be considered as 1 episode of infection if the neonate was clinically ill (e.g., ongoing temperature instability; respiratory, cardio circulatory, or neurological symptoms not explained by other conditions; or ongoing laboratory abnormalities such as elevated C-reactive protein) and received antibiotic therapy for more than 5 days. The no infection group consisted of infants who had no culture-proven bacteremia and/or meningitis. Ventilator associated pneumonia, urinary tract infection, and necrotizing enterocolitis were classified as late onset CPBSI or late onset meningitis if they had a positive blood or cerebrospinal fluid culture as defined above; otherwise they were classified as no infection. By inference, early onset CPBSI was defined as at least 1 positive blood culture obtained from a neonate clinically suspected of having infection within 2 calendar days of life. Similarly, early onset meningitis was defined by at least 1 positive cerebrospinal fluid culture obtained from a neonate clinically suspected of infection within 2 calendar days of life, with or without late onset CPBSI. IVH was defined according to the classification of Papile et al. [10]. Information on whether a cerebrospinal fluid reservoir or shunt procedure was performed was available but not the date of the procedure. Infant characteristics included GA, birth weight, small for GA (SGA), and sex. GA was calculated by in vitro fertilization date, last menstrual period, antenatal ultrasound examination, obstetrical estimate, or neonatal estimate (in that order). A neonate was classified as SGA if the birth weight was $<10$ th percentile as per GA and sex [11]. Postnatal variables included intensive resuscitation, 5-min Apgar score $<7$, and Score for Neonatal Acute Physiology version II (SNAP-II) score $>20$. Intensive resuscitation was defined as requiring any invasive ventilation, vasopressors, or chest compression. Admission severity of illness was characterized by the SNAP-II [12]. Prenatal and maternal variables included multiple births, mode of delivery, delivery outside of a tertiary NICU (outborn), antenatal steroid use (at least 1 dose $>12 \mathrm{~h}$ before delivery), maternal antibiotic use (any antibiotic exposure within $24 \mathrm{~h}$ before delivery), maternal diabetes, maternal hypertension, and maternal age.

\section{Outcomes}

The primary outcome was a multinomial outcome of infection category (no infection, late onset CPBSI only, and late onset meningitis). We treated IVH as an exposure since it generally occurs before late onset infections.

\section{Statistical Analysis}

The study population was summarized descriptively. The rates of late onset CPBSI and late onset meningitis were estimated for each admission year and the trends of their rates were examined using Cochran-Armitage trend tests. The slopes of the trends for late onset CPBSI and late onset meningitis were compared using linear regression model with interaction term between infection category and admission year. A significant in- 


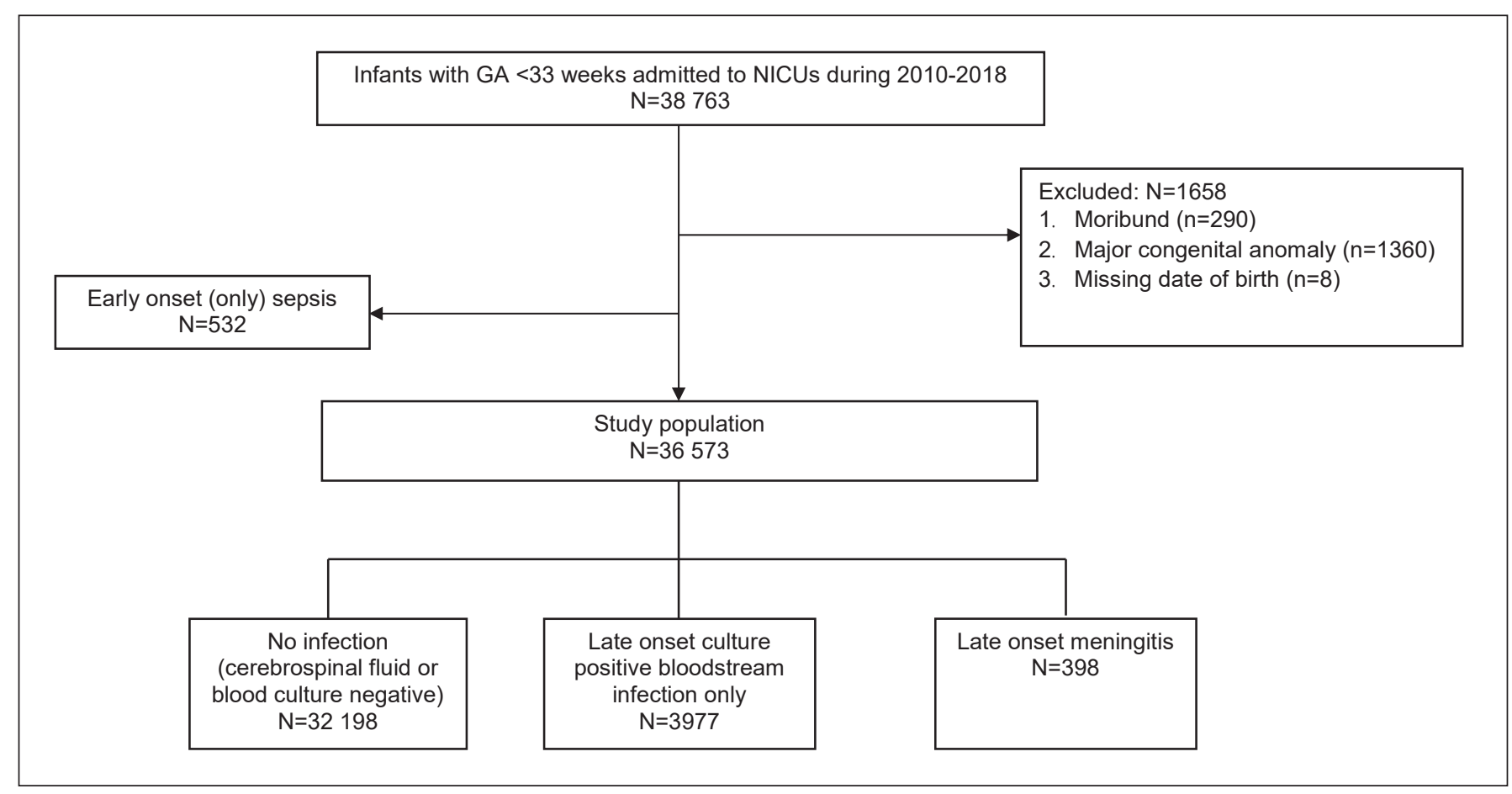

Fig. 1. Flowchart of infant enrollment in study. GA, gestational age; NICU, neonatal intensive care unit.

teraction term indicates that the slopes of the trends are significantly different. We also determined and compared the trends using multivariable multinomial logit regression model adjusted for potential confounders. Subgroup analyses for 22-28 and 2932 weeks GA infants were performed. To identify possible differences in characteristic risk factors for CPBSI and meningitis, the maternal and infant characteristics were first compared among the primary outcome's 3 categories using $\chi^{2}$ test for categorical variables, and $F$ test or Kruskal-Wallis test as appropriate for continuous variables. Pairwise comparisons were conducted to examine differences in characteristics between any 2 groups using $\chi^{2}$ test for categorical variables and Student's $t$ test or Wilcoxon Rank Sum test for continuous variables. A final multiple multinomial logit model was derived by backward variables selection procedure to determine the independent risk factors of the primary outcome. The covariates included in the full model were all potential risk factors identified in univariate analysis. To examine the association of IVH with meningitis, multivariable multinomial logit models adjusted for potential confounders were used. Subgroup analyses for 22-28 and 29-32 weeks GA infants were performed. Sensitivity analyses were performed to exclude infants (1) who had cerebrospinal fluid shunt or reservoir procedures, and (2) who had any infections occurring within 3 days of life or CoNS meningitis. Data management and statistical analyses were performed using SAS 9.4 (SAS Institute, Cary, NC, USA) and R 4.0.0 (www.r-project.org). Twosided $p$ value of $<0.05$ was considered statistically significant.

\section{Results}

Between 2010 and 2018, 38,763 preterm infants $<33$ weeks GA were admitted to CNN NICUs, and 36,573 were included in the study after applying exclusion criteria (Fig. 1). Of these, 32,198 had no infections, 3,977 had only late onset CPBSI, and 398 had late onset meningitis (with or without CPBSI). Forty seven infants with meningitis received a cerebrospinal fluid shunt or reservoir procedure. Data on types of organisms are described and presented graphically in the supplementary material (online suppl. Fig. 1; for all online suppl. material, see www. karger.com/doi/10.1159/000520424).

Figure 2 shows that there was a significant and consistent decreasing trend in the incidence of late onset CPBSI (slope $-0.62, p<0.001$ ) but no significant change in the incidence trend of late onset meningitis (slope -0.013 , $p=0.70$ ) between 2010 and 2018. The trends for late onset CPBSI and late onset meningitis were significantly different $(p<0.001)$. Multivariable analyses results showed that the findings remained significant after adjustment for gestational age, sex, small for gestational age, SNAP-II score, intensive resuscitation, Cesarean section, maternal diabetics, and maternal hypertension: the incidence of CPBSI decreased (adjusted odds ratio $[\mathrm{AOR}]=0.93 ; 95 \%$ 
Fig. 2. Trends in late onset meningitis and late onset CPBSI rates by admission year for eligible infants with GA $<33$ weeks. Late onset meningitis: slope $=-0.013, p=0.70$; late onset CPBSI: slope $=-0.62, p<0.001$. CPBSI, culture positive bloodstream infection; GA, gestational age.

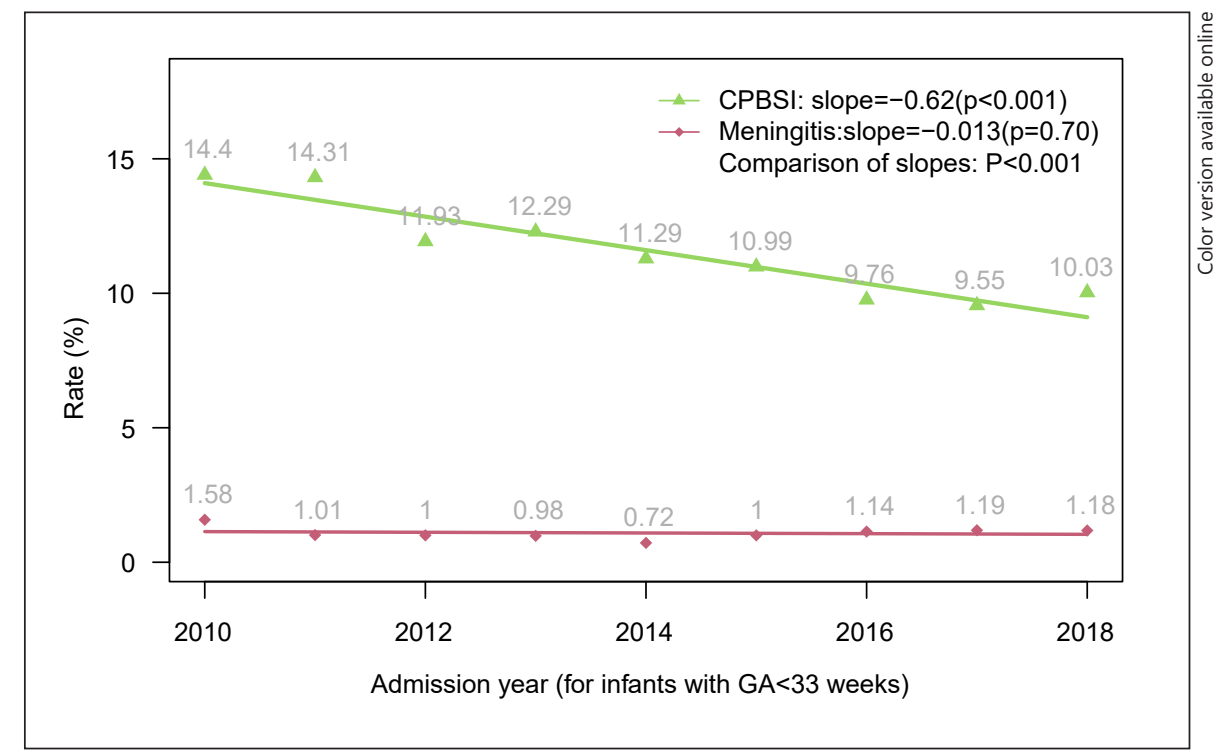

Table 1. Maternal and infant characteristics by infection category

\begin{tabular}{|c|c|c|c|c|}
\hline & \multicolumn{3}{|l|}{ Infection category } & \multirow[t]{2}{*}{$p$ value } \\
\hline & $\begin{array}{l}\text { no infection } \\
(n=32,198)\end{array}$ & $\begin{array}{l}\text { late onset CPBSI } \\
(n=3,977)\end{array}$ & $\begin{array}{l}\text { late onset } \\
\text { meningitis } \\
(n=398)\end{array}$ & \\
\hline \multicolumn{5}{|l|}{ Maternal characteristics } \\
\hline Maternal age, mean (SD), year & $31.1(5.9)^{\mathrm{a}}$ & $30.3(6.1)$ & $31.5(5.8)$ & 0.003 \\
\hline Hypertension, \% $(n / N)$ & $19.4(6,044 / 31,097)^{\mathrm{b}}$ & $18.5(708 / 3,833)^{c}$ & $9.2(36 / 391)$ & $<0.0001$ \\
\hline Antenatal steroid use, \% $(n / N)$ & $87.6(27,451 / 31,335)$ & $88.5(3,421 / 3,865)$ & $87.0(340 / 391)$ & 0.24 \\
\hline Maternal antibiotic use, $\%(n / N)$ & $70.3(21,300 / 30,314)^{b}$ & $71.4(2,655 / 3,721)^{c}$ & $77.0(295 / 383)$ & 0.007 \\
\hline Rupture of membranes $>24 \mathrm{~h}, \%(n / N)$ & $21.7(6,628 / 30,545)$ & $20.9(785 / 3,761)^{c}$ & $25.5(100 / 393)$ & 0.09 \\
\hline Cesarean section, $\%(n / N)$ & $60.5(19,404 / 32,085)^{b}$ & $59.3(2,355 / 3,969)^{c}$ & $48.2(192 / 398)$ & $<0.0001$ \\
\hline \multicolumn{5}{|l|}{ Infant characteristics } \\
\hline \multicolumn{5}{|l|}{ GA group, \% (n/N) } \\
\hline Sex (male), $\%(n / N)$ & $54.8(17,607 / 32,158)^{a}$ & $57.1(2,270 / 3,973)$ & $54.3(216 / 398)$ & 0.017 \\
\hline $\mathrm{SGA}, \%(n / N)$ & $9.5(3,040 / 32,161)^{a}$ & $13.8(549 / 3,975)^{c}$ & $6.8(27 / 398)$ & $<0.0001$ \\
\hline Singleton, $\%(n / N)$ & $69.0(22,197 / 32,193)^{a}$ & $72.7(2,892 / 3,977)$ & $71.6(285 / 398)$ & $<0.0001$ \\
\hline Apgar score $<7$ at $5 \mathrm{~min}, \%(n / N)$ & $25.0(7,931 / 31,716)^{a, b}$ & $42.6(1,659 / 3,898)$ & $46.1(181 / 393)$ & $<0.0001$ \\
\hline SNAP-II score $>20, \%(n / N)$ & $12.6(4,018 / 31,814)^{a, b}$ & $29.3(1,160 / 3,964)^{c}$ & $36.9(146 / 396)$ & $<0.0001$ \\
\hline Outborn, \% $(n / N)$ & $15.2(4,894 / 32,178)$ & $14.7(584 / 3,977)$ & $13.6(54 / 398)$ & 0.47 \\
\hline Intensive resuscitation at birth, \% $(n / N)$ & $26.4(8,367 / 31,740)^{a, b}$ & $54.4(2,133 / 3,920)$ & $54.2(213 / 393)$ & $<0.0001$ \\
\hline
\end{tabular}

The reported $p$ values were based on the comparisons among 3 groups using $x^{2}$ test for categorical variables and $F$ test for continuous variables. Pairwise comparisons were also conducted and reported when significant $(p<0.05)$ using $x^{2}$ test for categorical variables and Student's $t$ test for continuous variables. CPBSI, culture positive bloodstream infection; SD, standard deviation; SNAP, Score for Neonatal Acute Physiology. ${ }^{\mathrm{a}} p<0.05$ No infection versus Late onset CPBSI. ${ }^{\mathrm{b}} p<0.05$ No infection versus Late onset meningitis. ${ }^{\mathrm{C}} p<0.05$ Late onset CPBSI versus Late onset meningitis.

Trends of Late Onset Culture Positive Bloodstream Infections and Meningitis
Neonatology 2022;119:60-67 DOI: $10.1159 / 000520424$ 
Table 2. Multivariable multinomial logistic regression results for maternal and infant characteristics independently predictive of late onset CPBSI, late onset meningitis, and comparison of late onset meningitis with late onset CPBSI

\begin{tabular}{|c|c|c|c|}
\hline & $\begin{array}{l}\text { Late onset CPBSI } \\
\text { versus no infection: } \\
\text { AOR }^{\mathrm{a}}(95 \% \mathrm{Cl})\end{array}$ & $\begin{array}{l}\text { Late onset meningitis } \\
\text { versus no infection: } \\
\text { AOR }(95 \% \mathrm{Cl})\end{array}$ & $\begin{array}{l}\text { Late onset meningitis } \\
\text { versus late onset CPBSI } \\
\text { AOR }(95 \% \mathrm{Cl})\end{array}$ \\
\hline \multicolumn{4}{|l|}{ Maternal characteristics } \\
\hline Diabetes & $0.79(0.69,0.89)^{b}$ & $0.90(0.62,1.29)$ & $1.14(0.78,1.67)$ \\
\hline Hypertension & $1.11(0.99,1.24)$ & $0.67(0.46,0.98)^{\mathrm{b}}$ & $0.60(0.41,0.89)^{\mathrm{b}}$ \\
\hline Caesarean section & $0.95(0.87,1.03)$ & $0.67(0.54,0.84)^{\mathrm{b}}$ & $0.71(0.56,0.9)^{\mathrm{b}}$ \\
\hline \multicolumn{4}{|l|}{ Infant characteristics } \\
\hline GA: $29-32$ versus $22-28$ weeks & $0.19(0.18,0.21)^{\mathrm{b}}$ & $0.20(0.15,0.27)^{b}$ & $1.04(0.77,1.39)$ \\
\hline Male & $1.15(1.06,1.24)^{b}$ & $1.06(0.85,1.31)$ & $0.92(0.74,1.16)$ \\
\hline SGA & $1.68(1.48,1.90)^{b}$ & $1.16(0.75,1.79)$ & $0.69(0.44,1.08)$ \\
\hline Apgar score $<7$ at $5 \mathrm{~min}$ & $1.12(1.03,1.22)^{\mathrm{b}}$ & $1.32(1.04,1.69)^{\mathrm{b}}$ & $1.18(0.92,1.53)$ \\
\hline SNAP-II score $>20$ & $1.28(1.16,1.40)^{\mathrm{b}}$ & $1.86(1.45,2.38)^{\mathrm{b}}$ & $1.45(1.13,1.88)^{\mathrm{b}}$ \\
\hline Intensive resuscitation at birth & $1.65(1.51,1.80)^{\mathrm{b}}$ & $1.37(1.06,1.77)^{\mathrm{b}}$ & $0.83(0.64,1.09)$ \\
\hline
\end{tabular}

AOR, adjusted odds ratio, for example, odds of late onset CPBSI versus no Infection = Prob (late onset CPBSI)/ Prob(no infection); $\mathrm{Cl}$, confidence interval; $\mathrm{CPBSI}$, culture positive bloodstream infection; SNAP, Score for Neonatal Acute Physiology; GA, gestational age; SGA, small for gestational age a $\mathrm{AOR}(95 \% \mathrm{Cl})$ of the outcome were determined based on the final multinomial logistic regression model derived by backward variable selection procedure. The covariates included in the full model were those identified associated with the outcome in the univariate analyses. Birth weight was not included, to avoid the multi-collinearity. ${ }^{\mathrm{b}} p<0.05$.

Table 3. Association of intraventricular hemorrhage rates with late onset $\mathrm{CPBI}$ and late onset meningitis

\begin{tabular}{|c|c|c|c|c|}
\hline & \multicolumn{4}{|l|}{ Infection category } \\
\hline & no infection & late onset CPBSI only & late onset meningitis & $p$ value \\
\hline No. infants & 32,198 & 3,977 & 398 & \\
\hline IVH grade 1 or $2, \%(n / N)$ & $20.2(5,110 / 25,293)^{a, b}$ & $30.99(1,189 / 3,837)$ & $31.36(122 / 389)$ & $<0.0001$ \\
\hline IVH grade 3 or higher, $\%(n / N)$ & $4.9(1,241 / 25,293)^{a, b}$ & $9.3(357 / 3,837)^{c}$ & $27.8(108 / 389)$ & $<0.0001$ \\
\hline
\end{tabular}

The reported $p$ values were based on the comparisons among 3 groups using $x^{2}$ test. Pairwise comparisons were also conducted and reported when significant $(p<0.05)$ using $x^{2}$ test. CPBSI, culture positive bloodstream infection; $\mathrm{IVH}$, intraventricular hemorrhage. ${ }^{\mathrm{a}} p<0.05$ No infection versus late onset CPBSI. ${ }^{\mathrm{b}} p<0.05$ No infection versus late onset meningitis. ${ }^{c} p<0.05$ Late onset CPBSI versus late onset meningitis.

confidence interval [CI] 0.92, 0.95), while the incidence of meningitis was unchanged $(\mathrm{AOR}=0.98 ; 95 \% \mathrm{CI} 0.94$, 1.01). The trends for CPBSI and meningitis were significantly different $(p=0.04)$. The results remained robust when subgroup analyses for 22-28 and 29-32 weeks GA infants were performed (see online suppl. Fig. 2; online suppl. Table 1).

Table 1 compares maternal and infant characteristics between the no infection, late onset CPBSI, and late onset meningitis groups. Table 2 shows the independent risk factors associated with late onset CPBSI or late onset meningitis based on multivariable multinomial logit regression analyses.
The incidence of severe IVH (grade 3 or above) was significantly higher (Table 3 ) in infants with late onset meningitis (27.8\%) than in infants with late onset CPBSI (9.3\%) and infants with no infection (4.9\%). The median (IQR) age of occurrence of late onset CPBSI and late onset meningitis were $14(9,24)$ days and $18(10,40)$ days, respectively. Since IVH mostly occurs earlier than late onset meningitis, we examined the potential association of IVH and meningitis using multivariable multinomial logit models (Table 4). Compared to infants with no IVH grade 3 or above, infants with IVH grade 3 or above had higher odds of late onset meningitis versus no infection (AOR 4.16; 95\% CI 3.17, 5.44), and higher odds of late
64 
Table 4. Multinomial logistic regression showing associations of IVH and infection categories

\begin{tabular}{|c|c|c|c|}
\hline Exposure & $\begin{array}{l}\text { AOR }(95 \% \mathrm{Cl}) \text { : late onset } \\
\text { CPBSI versus no } \\
\text { infection }\end{array}$ & $\begin{array}{l}\text { AOR ( } 95 \% \mathrm{Cl}) \text { : late onset } \\
\text { meningitis versus no } \\
\text { infection }\end{array}$ & $\begin{array}{l}\text { AOR }(95 \% \mathrm{Cl}) \text { : late onset } \\
\text { meningitis versus late } \\
\text { onset CPBSI }\end{array}$ \\
\hline IVH (grade 1 or 2 ) & $1.25(1.14,1.37)^{c}$ & $1.25(0.98,1.59)$ & $1.00(0.78,1.28)$ \\
\hline IVH (grade 3 or above) & $1.01(0.87,1.17)$ & $4.16(3.17,5.44)^{c}$ & $4.11(3.08,5.50)^{c}$ \\
\hline \multicolumn{4}{|l|}{ Sensitivity analysis $\mathrm{I}^{\mathrm{a}}$} \\
\hline IVH (grade 1 or 2 ) & $1.26(1.15,1.37)^{c}$ & $1.46(1.14,1.87)^{c}$ & $1.16(0.89,1.50)$ \\
\hline IVH (grade 3 or above) & $0.99(0.86,1.15)$ & $2.62(1.92,3.56)^{c}$ & $2.64(1.90,3.65)^{c}$ \\
\hline \multicolumn{4}{|l|}{ Sensitivity analysis II } \\
\hline IVH (grade 1 or 2 ) & $1.25(1.15,1.37)^{c}$ & $1.43(1.06,1.95)^{c}$ & $1.15(0.84,1.56)$ \\
\hline IVH (grade 3 or above) & $0.99(0.85,1.15)$ & $3.14(2.17,4.55)^{c}$ & $3.19(2.16,4.70)^{c}$ \\
\hline
\end{tabular}

AOR, adjusted odds ratio; $\mathrm{Cl}$, confidence interval; CPBSI, culture positive bloodstream infection; IVH,

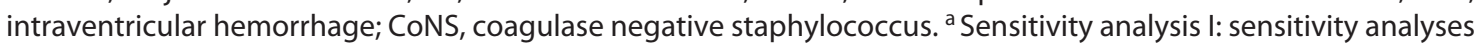

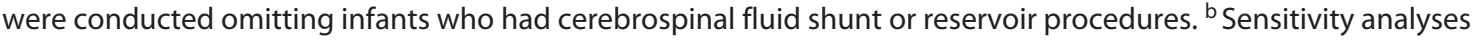
II: sensitivity analysis were conducted where late onset infection was defined as infection after 3 days of birth and CoNS meningitis were excluded. ${ }^{c} p<0.05$.

onset meningitis versus late onset CPBSI (AOR 4.11;95\% CI 3.08, 5.50) (see online suppl. explanatory note for Table 4). The results remained robust when subgroup analyses for 22-28 and 29-32 weeks GA infants were performed (see online suppl. Table 2 ). In sensitivity analyses, the results remained robust when infants who had cerebrospinal fluid shunt or reservoir procedures were excluded (Table 4). The results also remained robust when infants with infections identified within 3 calendar days were excluded, and when CoNS-related meningitis were excluded. Data for those with and without CSF hardware (shunt or reservoir) were presented in online suppl. Table 3.

\section{Discussion}

To our knowledge, this is the first report that there is a decreasing trend for late onset CPBSI but not for late onset meningitis. This observation is important because it suggests that their risk factors may be different, which may have implications for prevention strategies. Risk factors for late onset nosocomial infection in preterm infants $<33$ weeks are well documented, and include perinatal and obstetric risks, diminishing maternal antibodies, immunological immaturity, colonization by pathogenic organisms, exposure to antibiotics, malnutrition, skin breaks and invasive procedures, associated illnesses, and prolonged hospitalization [13]. Consequently, infection prevention strategies have focused on minimizing these

Trends of Late Onset Culture Positive

Bloodstream Infections and Meningitis risks and quality improvement strategies based on these approaches have been variably successful at reducing late onset nosocomial infections [5]. In a cluster randomized controlled trial of 12 Canadian NICUs using a quality improvement process from 2003 to 2005, Lee et al. [14] demonstrated $44 \%$ reduction in the rates of nosocomial infection. Similarly, Bowen et al. [15] reported $>50 \%$ reduction in bloodstream infections in extremely preterm neonates following a collaborative quality improvement project to reduce neonatal infection in an Australian NICU network. We found similar reduction of late onset CPBSI but not meningitis in our study, which occurred at the same time as a national NICU quality improvement initiative in Canada [5]. It is possible that the divergence in trends of late onset CPBSI and late onset meningitis in our study is because of the small numbers of patients with meningitis. However, the results are statistically significant and persist despite adjustment for GA and other risk factors, suggesting that further examination of the risk differences between late onset CPBSI and meningitis may be warranted.

We also observed that severe IVH is associated with late onset meningitis even after exclusion of infants requiring CSF shunt or reservoir. While this may be because of mechanisms not evaluated in this study, it is also possible that there is a pathophysiological relationship between the 2. Risk factors described for meningitis include hematogenous spread, direct extension from an infectious foci, and tissue disruptions from trauma or congenital defects [16]. Mechanisms for bacterial entry to 
the cerebrospinal fluid space include transcellular movement across the endothelial cell (e.g., Group B streptococcus), paracellular movement by disruption of intercellular tight junctions (e.g., enteric pathogens), and transport across the blood-brain barrier and blood-cerebrospinal fluid barriers within phagocytes (e.g., Listeria monocytogenes) [17-19], and the presence of intraventricular devices. The presence of bacteria induces release of inflammatory mediators into the cerebrospinal fluid, resulting in meningeal inflammation and increased permeability of the blood-brain barrier [17, 20]. Leviton et al. [6] reported that IVH was associated with elevated levels of systemic inflammation, including CRP, IL-8, SAA, TNF-alpha, and others, and that the presence of white matter injury resulted in an even stronger signal. They postulated that IVH may promote the upregulation of inflammatory proteins and the risk of excitotoxicity damage and white matter injury, or that the inflammation stimulus promotes both IVH and white matter injury. Blood in the ventricle may also stretch and disrupt the ependymal barrier allowing inflammatory contents in the cerebrospinal fluid access to the surrounding parenchyma and causing injury. Since the inflammatory pathway features prominently in both meningitis and intraventricular hemorrhage, it is possible that the inflammatory response triggered by IVH may facilitate bacterial penetration into the cerebrospinal fluid space and leading to bacterial meningitis [7]. The higher risk of late onset meningitis with grade 3 or higher IVH than grade 1 or 2 IVH may also suggest a dose effect of inflammation. Whether the concurrent occurrence of IVH and bacterial meningitis further amplifies tissue damage merits further research. Whether the inflammatory response induced by IVH also impacts on other organs is of interest, and further research is needed to determine the potential implications for BPD, ROP, NEC, and brain development.

Prevention of IVH is an area of active research and Murthy et al. [21] recently demonstrated 69\% reduction in risk of severe brain injury using a neuroprotective bundle that included optimization of antenatal and perinatal management (including delivery in appropriate facilities, antenatal corticosteroid administration, delayed cord clamping, and obstetric management), skilled resuscitation, circulatory support to avoid hypotension and fluctuating arterial blood pressure, respiratory support to avoid hypoxia, hypercarbia and acidosis, correction of coagulation abnormalities, minimal handling and stimulation after birth, and midline positioning of the infant head [21]. It would be instructive to see if IVH re- duction also resulted in a reduction of late onset meningitis.

Limitations of this study include lack of more granular information on clinical treatment that may be useful for identifying therapy-related risk factors for late onset meningitis and developing prevention strategies; and lack of information about inflammatory markers in relation to onset of meningitis, which may provide insights into the pathophysiology of the inflammation-meningitis pathways. Since the definition of late onset CPBSI and late onset meningitis in this study is after 2 calendar days, it is still within the window for IVH to occur. In addition, we did not have information on whether insertion of cerebrospinal fluid shunts and reservoirs preceded the occurrence of meningitis. However, our results remained robust when a sensitivity analysis omitting all infants who had a cerebrospinal fluid shunt or reservoir, was conducted. In addition, since some authors [22] defined late onset infections as occurring after 3 days of life and excluded CoNS meningitis because it may be a contaminant, we also performed sensitivity analyses excluding these infants and our results remained robust. We did not exclude CoNS CPBSI because this is a recognized cause of sepsis in preterm infants. We did not have information on repeated lumbar punctures.

\section{Conclusions}

In summary, we found that there was a decreasing trend of late onset CPBSI but not late onset meningitis among preterm infants $<33$ weeks GA in Canadian NICUs; and there was an association between late onset meningitis and IVH. Further research is needed to identify risk factors for late onset meningitis that may be different from late onset CPBSI and to develop strategies for reducing late onset meningitis.

\section{Acknowledgments}

The authors gratefully acknowledge the work of all site investigators, data abstractors, and trainers of the Canadian Neonatal Network (CNN). We thank the staff at the Maternal-infant Care Research Center (MiCare) at Mount Sinai Hospital in Toronto, $\mathrm{ON}$, Canada, for organizational support of CNN. In particular, we thank Heather McDonald Kinkaid, $\mathrm{PhD}$, a medical writer and editor and full-time employee receiving a salary from MiCare, for editorial assistance in formatting this manuscript for submission.
Zhou et al. 


\section{Statement of Ethics}

CNN has waiver of informed consent from ethics. Ethical approval for this study was obtained from the Research Ethics Board at Mount Sinai Hospital and the Executive Committee of the CNN, with the Reference Number REB \#19-0267-C.

\section{Conflict of Interest Statement}

The authors have no conflicts of interest to declare.

\section{Funding Sources}

Organizational support for the Canadian Neonatal Network was provided by the Maternal-infant Care Research Center (MiCare) at Mount Sinai Hospital in Toronto, ON, Canada. MiCare is supported by the Canadian Institutes of Health Research (CIHR) (CTP 87518), the Ontario Ministry of Health and Long-Term
Care, and the participating hospitals. PSS holds an Applied Research Chair in Reproductive and Child Health Services and Policy Research from the CIHR (APR-126340).

\section{Author Contributions}

Q.Z. and S.K.L. contributed to the conception of the research; all authors contributed to the design of the research; M.O., M.L., and X.Y.Y. contributed to the acquisition and analysis of the data; all authors contributed to the interpretation of the data; and Z.Q. drafted the manuscript. P.S.S., J.Y.T., and S.K.L. critically revised the manuscript, and all authors approved the final manuscript.

\section{Data Availability Statement}

All data generated or analyzed during this study are included in this. Further inquiries can be directed to the corresponding author.

\section{References}

1 Schlapbach LJ, Aebischer M, Adams M, Natalucci G, Bonhoeffer J, Latzin P, et al. Impact of sepsis on neurodevelopmental outcome in a Swiss National Cohort of extremely premature infants. Pediatrics. 2011;128(2):e348-57.

2 Seale AC, Head MG, Fitchett EJ, Vergnano S, Saha SK, Heath PT, et al. Neonatal infection: a major burden with minimal funding. Lancet Glob Health. 2015;3(11):e669-70.

3 Collins A, Weitkamp JH, Wynn JL. Why are preterm newborns at increased risk of infection? Arch Dis Child Fetal Neonatal Ed. 2018; 103(4):F391-4.

4 Lee SK, Shah PS, Singhal N, Aziz K, Synnes A, McMillan D, et al. Association of a quality improvement program with neonatal outcomes in extremely preterm infants: a prospective cohort study. CMAJ. 2014;186(13):E485-94.

5 Lee SK, Beltempo M, McMillan DD, Seshia $M$, Singhal N, Dow K, et al. Outcomes and care practices for preterm infants born at less than 33 weeks' gestation: a quality-improvement study. CMAJ. 2020;192(4):E81-91.

6 Leviton A, Allred EN, Dammann O, Engelke S, Fichorova RN, Hirtz D, et al. Systemic inflammation, intraventricular hemorrhage, and white matter injury. J Child Neurol. 2013; 28(12):1637-45.

7 van Furth AM, Roord JJ, van Furth R. Roles of proinflammatory and anti-inflammatory cytokines in pathophysiology of bacterial meningitis and effect of adjunctive therapy. Infect Immun. 1996;64(12):4883-90.
8 Shah PS, Seidlitz W, Chan P, Yeh S, Musrap $\mathrm{N}$, Lee SK. Internal audit of the Canadian neonatal network data collection system. Am J Perinatol. 2017;34(12):1241-9.

9 Canadian Neonatal Network. Abstarctor's manual v3.5.0. 2021. Canadian Neonatal Network; 2021. Available from: http://www.canadianneonatalnetwork.org/portal/Portals/0/CNN\%20Manuals/CNN\%20Manual_20210225.pdf.

10 Papile LA, Munsick-Bruno G, Schaefer A. Relationship of cerebral intraventricular hemorrhage and early childhood neurologic handicaps. J Pediatr. 1983;103(2):273-7.

11 Kramer MS, Platt RW, Wen SW, Joseph KS, Allen A, Abrahamowicz M, et al. A new and improved population-based Canadian reference for birth weight for gestational age. Pediatrics. 2001;108(2):E35.

12 Richardson DK, Corcoran JD, Escobar GJ, Lee SK. SNAP-II and SNAPPE-II: simplified newborn illness severity and mortality risk scores. J Pediatr. 2001;138(1):92-100.

13 Downey LC, Smith PB, Benjamin DK. Risk factors and prevention of late-onset sepsis in premature infants. Early Hum Dev. 2010; 86(Suppl 1):7-12.

14 Lee SK, Aziz K, Singhal N, Cronin CM, James A, Lee DS, et al. Improving the quality of care for infants: a cluster randomized controlled trial. CMAJ. 2009;181(8):469-76.
15 Bowen JR, Callander I, Richards R, Lindrea $\mathrm{KB}$. Decreasing infection in neonatal intensive care units through quality improvement. Arch Dis Child Fetal Neonatal Ed. 2017; 102(1):F51-7.

16 Zainel A, Mitchell H, Sadarangani M. Bacterial meningitis in children: neurological complications, associated risk factors, and prevention. Microorganisms. 2021;9(3):535.

17 Polin RA, Harris MC. Neonatal bacterial meningitis. Semin Neonatol. 2001;6(2):15772.

18 Barichello T, Fagundes GD, Generoso JS, Elias SG, Simões LR, Teixeira AL. Pathophysiology of neonatal acute bacterial meningitis. J Med Microbiol. 2013;62(Pt 12):1781-9.

19 Kim KS. Acute bacterial meningitis in infants and children. Lancet Infect Dis. 2010;10(1) $32-42$.

20 Pong A, Bradley JS. Bacterial meningitis and the newborn infant. Infect Dis Clin North Am. 1999;13(3):711-viii.

21 Murthy P, Zein H, Thomas S, Scott JN, Abou Mehrem A, Esser MJ, et al. Neuroprotection care bundle implementation to decrease acute brain injury in preterm infants. Pediatr Neurol. 2020;110:42-8.

22 El-Naggar W, Afifi J, McMillan D, Toye J, Ting J, Yoon EW, et al. Epidemiology of meningitis in Canadian neonatal intensive care units. Pediatr Infect Dis J. 2019;38(5):476-80. 